\title{
Was dürfen wir hoffen?
}

Es gibt eine scheinbar paradoxe Doppeltendenz zwischen dem Wunsch, zu wissen, wie ein Film oder eine Serie endet, und dem Unbehagen, dass der Ausgang des Endes schon zwei Tage zuvor absehbar ist, wie zum Beispiel in der Serie Game of Thrones. Es geht um dieses Schwanken zwischen dem Wunsch nach der spannenden Erzählung und nach dem Ergebnis, durch das der Sinn vollendet wird und das uns überrascht. Unsere Freunde sollen auf keinen Fall das Ende »spoilern«. Dabei entsteht eine Spannung mit der Begierde, einen Ort zu erleben, an dem der Sinn rekonstruiert wird. Endlich ausbrechen aus den Inkohärenzen der Realität, auch wenn nur in eine Welt aus ausgedachten Szenen.

Wir suchen in fiktionalen Geschichten den in der Sozialgeschichte verlorengegangenen Sinn. Die gelebte Geschichte stellt uns jedoch an einen Wendepunkt zwischen dem Wunsch, das Unerträgliche hätte einen positiven Ausgang und dem Lernprozess, denjenigen zu misstrauen, die uns so etwas (nicht nur im Wahlkampf) versprechen. Die Politiker sprechen weiterhin, vielleicht als Regierungspartei davon, dass wir Transformationen durchleben, die Ähnlichkeiten mit der nationalen Unabhängigkeit oder mit einer entfernt scheinenden Revolution im eigenen Land hätten, oder sie sagen, wir würden eine sichere öffentliche Ordnung zurückgewinnen und dann würde der Wohlstand kommen. Unterdessen nehmen wir als Bürger Regierungen wahr, die es nicht schaffen, die Unsicherheit zu bewältigen, die von der Globalisierung, der wirtschaftlichen Prekarität, der Gewalt in Städten und den interkulturellen Konflikten im Zusammenhang von Migration, dem Generationenkonflikt und dem Verhältnis zwischen Verbrauchern und Nutzern mit Unternehmen ausgeht. Die Führungspersönlichkeiten, die die meiste Überzeugung vermitteln, sind diejenigen, die entglobalisierende Bewegungen fördern und es befürworten, sich »in sich selbst « zurückzuziehen, auch wenn ihre Macht oft mehr in der Zuneigung $\mathrm{zu}$ ihnen und der Nation liegt, als in informierten und begründeten Ansichten. In den Oppositionskräften finden wir noch mehr emotionale Appelle, anstatt einem rationalen Verständnis der Widersprüche: die Rechten rufen dazu auf, die Autorität wiederherzustellen oder direkt in den Autoritarismus überzugehen; unter den Linken versprechen viele das $\mathrm{Pa}$ radies, indem sie diejenigen angreifen wollen, die versuchen, die Diagnose $\mathrm{zu}$ erneuern und alternative Programme aufzubauen, die im globalen und regionalen Rahmen anwendbar sind. 
Diese Horizonte des Unbehagens werden immer trüber, wenn unbeherrschbare Auswirkungen der Algorithmen das Gefühl erzeugen, eine Welt ohne gemeinsam aufgehende Rechnung und ohne ein uns vereinendes Narrativ zu bewohnen. Für diesen Epilog besteht eine Herausforderung in der Akzeptanz, dass es keinen möglichen Spoiler gibt: Es ist schwierig, unbegrenzt so weiterzumachen, ohne zu wissen, was man tun soll und während man den Wunsch hat, Bürger zu sein.

Trotz allem sollten die Fortschritte in den Sektoren hervorgehoben werden, die sich von der Naivität um das globalisierende Epos vom Ende des 20. Jahrhunderts und von den Patentrezepten von Links, die nur zögerlich Diagnosen erneuern und ihre Misserfolge kaum infrage stellen, distanzieren. Welche Fortschritte sind das? Sie erscheinen im Besonderen, wenn die jungen Generationen die Analysen und die Versprechen des Liberalismus und der eingerosteten Linken ins Archiv verbannen, um sich stattdessen der aktuellen politischen, wirtschaftlichen und kommunikationsbezogenen Ökologie anzunehmen. Es geht nicht darum, die Krise des Kapitalismus in seiner elektronischen Phase zu de-historisieren, sondern um deren Situierung und die Wiederaufnahme der klassischen Kritiken im Sinne eines Archivs, das immer wieder konsultiert und studiert werden darf. Oder, wie Maurice Merleau-Ponty sagte, als er vor siebzig Jahren von den sowjetischen Konzentrationslagern erfuhr: der Marxismus bleibt weiterhin ein Ort, von dem aus man nachdenken kann.

Die gleiche Freiheit bei der Neuinterpretation ist notwendig in Bezug auf die fremdenfeindlichen, rassistischen, den vorglobalen Stolz wiederherstellenden neuen Rechtsextremen, die sich auch der fortschrittlichsten technologischen Ressourcen bedienen. Dies sind die ersten Schritte zur Erkenntnis über das, was wir hoffen dürfen. In beiden Fällen - bei der Erneuerung der Diagnose und der Begrenzung einer äußersten Bedrohung- sind die heute weit verbreitete Entfremdung der Politiker und die ungelösten Fragen der kritischen Positionen keine schlechten Nachrichten. Das unbequeme Leben ohne Spoiler führt dazu, dass viele das Akzeptieren der Unsicherheit mit einem negativen Gemüt verwechseln, oder dass wir beim Beenden einer Konferenz oder eines Buches aufgefordert werden, zu entscheiden, ob wir Pessimist oder Optimist seien. Ich sage da lieber, ich bin ein Anti-Skeptiker.

Es gibt drei grundlegende Aufgaben, um jetzt Bürger sein zu können: der Wiederaufbau eines undogmatischen und umfassenden Sinns der sozialen und weltweiten Heterogenitäten, der gegenwärtige Widersprüche, mit dem Zweck einer Solidarität auf eine andere Art und Weise; die Notwendigkeit 
zur Erkenntnis, dass wir etwas brauchen, auf das wir gemeinsam hoffen können; die Notwendigkeit zu wissen, dass der Inhalt dieser Hoffnung sowie die Möglichkeiten, dass sie in Erfüllung geht, ganz vielfältig sind, sich im Aufbau befinden und rationale und nachweisbare Überprüfungen erfordern.

Ich denke dabei etwa an die nachprüfbare Dokumentation von Verificado $19 S$ in Mexiko, oder der Inhalte in alternativen Gesellschaften im Kontext einer internationalen Gegeninformation wie Telegram, Cryptopunks oder der Software The Onion Router (TOR). Andere Beispiele sind die starken öffentlichen Proteste wie \#MeToo oder Ni una menos, die Wahrheit, Gerechtigkeit und Freiheit für ein gutes Leben fordern. Darüber hinaus fallen mir diskretere Besetzungen des öffentlichen Raums ein: Programme von Künstlergruppen und Kulturschaffenden zur Wiederbelebung urbaner Gebiete und der Schaffung von Partnerschaften und Netzwerken, um auf der Mikroebene regenerativ zu wirken. Es gibt viele Möglichkeiten, die Stadt zu gestalten und die »Wahrheiten« und ihre Interpretationen der Verbindungen mit anderen Formen der Globalisierung von unten selbst auszudrücken. Jenseits des Gegensatzes zwischen Domestizierung und Widerstand und über den Wunsch zum Aufbau unterschiedlicher Kulturpolitiken hinaus, schaffen diese Initiativen neue, produktive Staatsbürgerschaften. Es entstehen neue Formen des SichKennenlernens, des Sich-Kennens und des Ausdrucks.

Ich führe auf diesen Seiten das nachprüfbare Wissen, die feministischen Enthüllungen über sexuelle Belästigung und die Aktionen auf der Mikroebene, sowie die großen internationalen Festivals auf, weil diese Praktiken Alternativen bieten, die sich gegenseitig ergänzen. Sie bringen Glaubwürdigkeit, bewährtes Wissen und einen poetischen Sinn der individuellen und der kollektiven Subjektivität in die politische Arena und bieten so ein wiederhergestelltes Zusammenleben an, das wir folglich nicht nur den religiösen Bewegungen überlassen müssen. Wissenschaft und Kunst konvergieren - wie unzählige Vertreter beider Disziplinen zeigen - in einer Zeit, in der natürliche und soziale Strukturen mehr denn je modifizierbar sind und in der sogar die wichtigsten Kunstwerke verändert, Videos neu aufgenommen und Nachrichten durch Algorithmen geordnet werden. Die Traditionen, die sich die ganze Zeit über vermischen, machen den Fundamentalismus zu einer traurigen Nostalgie, zu einem Symptom der Angst vor der wissenschaftlichen Erkenntnis und Fakten oder vor anderen Formen, Geschichte zu erzählen und sich die Zukunft vorzustellen.

Oft wird ein Satz von Walter Benjamin zitiert: »Nur aus Liebe zu den Verzweifelten halten wir noch an der Hoffnung fest.« Wenn wir diesen Liebesta- 
ten mit einem gewissen Hang zum religiösen Mandat einen politischen Sinn verleihen wollen, ist es wichtig, sie mit Untersuchungen zu untermauern, die ihnen rationale und empirische Konsistenz geben. Sie müssen in einer Sinnstiftung münden, um die gemeinsamen Erfahrungen zwischen gewöhnlichen Bürgern, Forschern und Akademikern zu nähren.

Was wir hoffen dürfen, hängt von der gemeinsam gestalteten Veränderung $a b$, die Wege des Handelns und der Erkenntnis zu gestalten. Eines der größten Defizite ist aktuell in Lateinamerika bei der Verbindung von Politik und Wissenschaft die Mutlosigkeit der staatlichen Behörden gegenüber der wissenschaftlichen Forschung. Dabei ist auffällig, dass die Rolle, die politische Führer dem Staat und den Menschenrechten zuschreiben, sich trotz einiger Unterschiede deutlich ähnelt. Regierungen wie die unter Mauricio Macri, Jair Bolsonaro und Andrés Manuel López Obrador teilen trotz bestehender Unterschiede diese eine Verhaltensweise. Wie entkommen wir dieser Kette der Fehldiagnosen und der Frustration, wenn nicht durch das Fördern eines gründlichen und erneuerten Wissens? Hierzu muss gesagt werden, dass der Mangel an universal konsensfähigen Gesellschaftstheorien stattdessen den Rückgriff auf das Wissen des »weisen Volks« oder »unserer Leute« genau so wenig rechtfertigt, wie die Klugheit der Millennials, die durch die Ideologie der Kreativwirtschaft idealisiert wird. Diese Branche ist mit geisterhaften Entitäten vergleichbar, die Gruppen zusammenführen, die in ihrem Verhalten, ihrem Wissen oder ihren Intuitionen viel zu heterogen sind. Die missbräuchliche Verwendung dieser Begriffe für widersprüchliche Zwecke hat ihre Definition aufgebrochen und ihren Gebrauch teilweise sehr riskant gemacht. Aus diesem Grund ziehe ich den Begriff des Bürgers vor, da wir ihn, neben seiner wahlbezogenen und rechtlichen Bedeutung, im politischen und moralischen Kontext aufleben lassen können, und weil seine Verwendung und seine Kritik durch wissenschaftliche Analysen eingegrenzt werden können.

Die Wissenschaft kann sich auch irren, etwa wenn sie zu weit von den Lebensbedingungen und dem »anderen", nicht-wissenschaftlichen Wissen abrückt. Trotzdem verfügt sie über mehr Werkzeuge, um Konzepte zu schärfen, um den gesunden Menschenverstand und die Algorithmen mit komplexen Fragen zu konfrontieren, und um deren Folgen zu verstehen. Ohne diese Aufgaben, die eine verstärke Finanzierung für die Wissenschaft, die Technologien und die Geisteswissenschaft erfordern, werden wir die Richtung, in die sich unsere Gesellschaften (des-)organisieren oder (ent-)globalisieren, nicht ändern können. Sichtbar wird diese Tendenz anhand der Korrelation zwi- 
schen hohen Investitionen in die wissenschaftliche Forschung (2 bis $4 \%$ ) und dem sozioökonomischen Entwicklungs- und Lebensstandard in den OECDLändern, sowie an der offenkundigen Bewunderung für die Asiaten, wenn es um deren schnelles Wachstum, deren neue internationale Führungsrolle und die Überwindung der Armut geht. Was können wir uns von den lateinamerikanischen Ländern erhoffen, die wissenschaftlich das höchste Niveau vertreten (Argentinien, Brasilien, Kolumbien und Mexiko). Schon vor Jahrzehnten versprachen die Machthaber dort, $1 \%$ des BIP in die Forschung zu investieren - ohne zu liefern. Diese Vernachlässigung der wissenschaftlichen Entwicklung zu korrigieren ist nicht nur Sache von Spezialisten, die in den letzten Jahren aus Protest auf die Straßen von Buenos Aires, Rio de Janeiro, São Paulo oder Mexiko-Stadt gegangen sind, um sich gegen die massiven Kürzungen des Budgets zu wehren. Wie bei Entscheidungen über das Internet und über die Rolle der Öffentlichkeit in der Bildung, der Wissenschaft und der Gesundheitsversorgung wird diese Frage nun zu einer entscheidenden Verantwortung der Bürger, da sie ebenso wichtig für Lehrer, Wissenschaftler, Studierende, wie für verarmte Familien, Migranten und Arbeitnehmer ist.

\section{Was sind die Menschen?}

Aktuelle Trends stellen diese Frage mit unterschiedlichen Perspektiven. Wir haben intellektuelle Abhandlungen, wie die von Foucault, Lévi-Strauss und Harari gesehen, sowie die Überlegungen soziokultureller Bewegungen, die sozial beeinträchtigt werden und von Entbürgerlichung betroffen sind, wie in lateinamerikanischen Diktaturen, und extreme Konfrontationen mit den Grenzen des Menschlichen durch den Femizid, Leichenraub oder deren bewusstes Verschwindenlassen. Da manche diese Erfahrungen unerträglich finden, sprechen sie den Tätern ihre Menschlichkeit ab und fordern die Todesstrafe. Auf der anderen Seite, die manchmal mit der ersten Perspektive verbunden ist, kommt es durch die Entführung von Daten und der Entscheidungsmacht der Menschen, die von so vielen freiwillig über die Delegierung an algorithmische Systeme ausgeht, zu einem Verschwimmen der Identität und der Grenzen des Menschlichen, die durch die Modernität neu gesetzt werden.

Die Geschichte der kolonialen Eroberungen ist zeitgleich die Geschichte über die Zweifel an der eigenen Identität: von den anderen und denen, die kolonialisieren. Haben die Indios auch eine Seele? Nach Jahren sehen wir, dass 